\title{
MicroRNA-1285 inhibits malignant biological behaviors of human pancreatic cancer cells by negative regulation of YAP1
}

\author{
H. HUANG ${ }^{1, \ddagger}$, G. XIONG ${ }^{1, \neq}$, P. SHEN ${ }^{1}$, Z. CAO ${ }^{1}$, L. ZHENG ${ }^{2}$, T. ZHANG ${ }^{1, *}$, Y. ZHAO \\ ${ }^{1}$ Department of General Surgery, Peking Union Medical College Hospital, Chinese Academy of Medical Sciences and Peking Union Medical Col- \\ lege, Beijing, 100730, China; ${ }^{2}$ Department of Nuclear Medicine, Peking Union Medical College Hospital, Chinese Academy of Medical Sciences \\ and Peking Union Medical College, Beijing, 100730, China \\ *Correspondence: tpingzhang@yahoo.com \\ ${ }^{*}$ Contributed equally to this work.
}

Received May 30, 2016/ Accepted November 11, 2016

\begin{abstract}
Pancreatic ductal adenocarcinoma is a most deadly malignancy, with a 5 -year survival rate of $\sim 7 \%$. Chemotherapy is the main treatment strategy of this disease. However, the high rate of resistance to chemotherapeutic agent contributes to poor prognosis. MicroRNAs are essential for the initiation, progression and chemoresistance of human malignancies. Previous studies have shown that miRNA-1285 participates in renal cell carcinoma and hepatocellular carcinoma. However, its roles in pancreatic ductal adenocarcinoma are poorly understood. In this study, we confirmed that miR-1285 was significantly down-regulated in gemcitabine-resistant pancreatic cancer cell lines by qRT-PCR. We found that miR-1285 suppressed cell proliferation as well as increased the sensitivity of PDAC cells to gemcitabine by CCK8 assays in vitro. Results from transwell assay indicated that miR-1285 inhibited pancreatic cancer cell migration and invasion. Experiments using different cell lines got identical results. All those results demonstrated that miR-1285 act as tumor suppressor of pancreatic cancer. To our knowledge, this study is the first to elucidate the function of miR-1285 in pancreatic cancer. Western blotting analysis verified that miR-1285 negatively regulated YAP1 protein level, together with EGFR and $\beta$-catenin. YAP1 is a known oncoprotein of pancreatic cancer. As silencing of YAP1 activity might be beneficial in cancer prevention and treatment, our results suggest that miR-1285 might serve as a novel therapeutic target for miRNA-based therapy in pancreatic cancer. Further research elucidating the exact mechanisms of miRNA-1285 function and the correlation between miR-1285 levels in tissues or serum and clinical characteristics of pancreatic cancer is needed later.
\end{abstract}

Key words: pancreatic cancer, gemcitabine, chemoresistance, YAP1, miR-1285

Pancreatic ductal adenocarcinoma (PDAC) is a most deadly malignancy, with a 5 year survival rate of $\sim 7 \%[1]$. Complete surgical resection is the only potentially curative treatment of this malignancy. However, only $15-20 \%$ are eligible for surgery [2]. Chemotherapy is the main treatment strategy of this disease. Gemcitabine [3, 4], erlotinib [5] and nab-paclitaxel [6] are the only FDA-approved standard chemotherapy drugs for pancreatic cancer currently, which only produce a modest survival benefit in patients with advanced disease [7]. The high rate of resistance to chemotherapeutic agents partly contributes to the poor prognosis of patients with pancreatic cancer. Among patients with metastatic disease treated with gemcitabine, the 5-year survival rate is only $2 \%$, and 1 -year survival rate is only 17 to $23 \%$ [6]. Gemcitabine is the most frequently used chemotherapeutic drug, which is proved to improve quality of life and increase overall survival of patients [8]. However, 34.5\% of patients with metastatic pancreatic cancers had a progressive disease during gemcitabine treatment [9]. Thus, a better understanding of mechanisms and solution to chemoresistance may lead to new treatment strategies that improve the prognosis of this lethal disease.

In eukaryotes, miRNAs are 22 nucleotides in length. Recent work supports a role for microRNAs in the initiation, progression and chemoresistance of human malignancies [10]. The connections between microRNAs and chemoresistance have been the subject of much investigation in recent years. MicroRNAs may regulate chemosensitivity through regulating drug excretion, cell survival signal, cell cycle, cell apoptosis, EMT, cancer stem cell property, etc $[11,12]$. 
MiR-1285, also known as miR-1285-1, miR-1285-3p, is located in 7q21-q22. It is well established that miR-1285 can function as tumor suppressors in hepatocellular carcinoma [13] and renal carcinoma [14], while its roles in pancreatic cancer is unclear. In our study, we will mainly focus on the biological functions of miR-1285 as well as primary searching for its target genes.

The transcriptional co-activator Yes-associated protein 1 (YAP1) is a major effect factor of Hippo signal pathway, which takes an important part in organ development and tumorigenesis [15]. Nowadays, YAP1 is considered to participate in maintenance of malignant behavior of cancer [16], which provides a new direction of targeted drug development.

\section{Materials and methods}

Cell lines. The human pancreatic ductal adenocarcinoma (PDAC) cell lines AsPC-1, BxPC-3, MiaPaCa-2, PANC-1, SU86.86 and T3M4 cells were donated by Dr. Freiss $\mathrm{H}$ (University of Heidelberg, Heidelberg, Germany). AsPC-1, BxPC-3 and SU86.86 were maintained in RPMI 1640 (Hyclone Logan, UT, USA), MiaPaCa-2, PANC-1 and T3M4 cells were maintained in Dulbecco's Modified Eagle's Medium (DMEM, Hyclone). All Media were supplemented with $10 \%$ fetal bovine serum (FBS, Hyclone, Logan, UT, USA) at $37^{\circ} \mathrm{C}$ and $5 \% \mathrm{CO}_{2}$. AsPC-1-Gem and MiaPaCa-2-Gem are gemcitabine-resistance AsPC-1 and MiaPaCa-2 cells, which were obtained discontinuously by gradually increasing doses of gemcitabine by researchers in our lab. Both of them could survive and proliferate in medium with concentration of gemcitabine of $10 \mathrm{mM}$.

Oligonucleotide transfection. T3M4, SU86.86 and MiaPaCa-2 cells were chosen for further studies. They were transfected with oligonucleotides packaged by Lipofectamine 2000 (Invitrogen, Carlsbad, CA, USA) in 6-well plates $\left(5 \times 10^{5}\right.$ cells/well). MiR-1285 mimics, anti-miR-1285 together and matched controls were synthesized by Genepharma (Shanghai, China). The oligonucleotides were dissolved according to the Instructions. 1:1 mix oligonucleotide solutions and Lipofectamine 2000 followed by incubating which on ice for 20 minutes. Add 10ul mixed solutions to each well for transfection. All the steps were carried out according to the protocol provided by the manufacturer of Lipofectamine 2000. The sequences transfected are as follows:

MiR-1285 mimics sense (5'-3') UCUGGGCAACAAAGUGAGACCU;

anti-sense (5'-3') GUCUCACUUUGUUGCCCAGAUU; Mimics control sense (5'-3') UUCUCCGAACGUGUCACGUTT;

anti-sense (5'-3') ACGUGACACGUUCGGAGAATT; MiR-1285 inhibitor sense (5'-3') AGGUCUCACUUUGUUGCCCAGA;

Inhibitor control sense (5'-3') CAGUACUUUUGUGUAGUACAA
RNA extraction and real-time quantitative reversetranscription-PCR (qRT-PCR). PDAC cells were transfected in 6-well plates $\left(5 \times 10^{5}\right.$ cells/well) for 48 hours. Total RNA was extracted using TRIzol reagent (Invitrogen, Carlsbad, CA). The quality of the RNA was evaluated using NanoDrop ND-1000 spectrophotometer (NanoDrop Technologies, USA) at the wavelengths of 260 and 280nm (A260/280). Complementary DNA was synthesized with TaqMan MicroRNA RT Kit (Applied Biosystems) and the reverse transcription kit (Promega, Madison, WI) for miRNAs and mRNAs, respectively. Quantitative RT-PCR (qRT-PCR) was performed using TaqMan MicroRNA Assays (Applied Biosystems) and SYBR Green Master Mix (Takara, Japan). U6 RNA and GAPDH were chosen as internal control for detection of mRNA and miRNA, respectively. Specific miRNA primers were synthesized by Applied Biosystems. The relative expression of miR-1285 and mRNAs were calculated depending on the $2^{-\Delta \Delta C T}$ methods. The primer sequence are as followed:

YAP1 sense (5'-3') CAGCAACTGCAGATGGAGAA; anti-sense (5'-3') ACATCCCGGGAGAAGACACT.

GAPDH sense (5'-3') CGGAGTCAACGGATTTGGTCGTAT; anti-sense (5'-3') AGCCTTCTCCATGGTGGTGAAGAC.

Cell proliferation assay. PDAC cells were triplicately planted into 96 -well culture plate(1000 cells/well) $24 \mathrm{~h}$ after transfection, in medium supplemented with 10\% FBS. All the plates were cultured at $37^{\circ} \mathrm{C}$ and $5 \% \mathrm{CO}_{2} .10 \mu \mathrm{L} /$ well cell count kit (CCK-8) reagent was added on $0 \mathrm{~h}, 24 \mathrm{~h}, 48 \mathrm{~h}$ and $72 \mathrm{~h}$ after planting. Optical densities at a wavelength of $450 \mathrm{~nm}$ (OD450) were measured after an additional 2.5 hours of CCK-8 incubation period at $37^{\circ} \mathrm{C}$ using a microplate reader (Wellscan MK3, Thermo/Labsystems, Finland). OD630 were served as a reference.

Growth inhibition assay. PDAC cells were triplicately planted into 96 -well culture plate(4000 cells/well) $24 \mathrm{~h}$ after transfection. After incubation at $37^{\circ} \mathrm{C}$ and $5 \% \mathrm{CO}_{2}$ for $4-6$ hours for cell adherence, gemcitabine (Eli Lilly and Company) of concentration gradient from $100 \mathrm{nM}$ to $1 \mathrm{mM}$ was added into each well. Ten $\mu \mathrm{L} /$ well cell count kit (CCK-8) reagent was added after incubation at $37^{\circ} \mathrm{C}$ for an extra $48 \mathrm{~h}$. Optical densities at a wavelength of $450 \mathrm{~nm}$ (OD450) were measured after an additional 2.5 hours of CCK-8 incubation period at $37{ }^{\circ} \mathrm{C}$ using a microplate reader (Wellscan MK3, Thermo/ Labsystems, Finland). OD630 were served as a reference.

Cell migration and invasion. Cell migration assays were performed in triplicate using Transwell migration chambers ( $8 \mu \mathrm{m}$ pore size; Corning, USA). Invasion assays were coated with diluted ECM solution (Sigma-Aldrich, Shanghai, China) as described in the manufacturer's protocol. Cells were transfected with miR-1285 mimics, inhibitor or paired control oligonucleotides, cultured for $48 \mathrm{~h}$, and then transferred on the top of upper chambers or ECM gel in a serum-free culture $\left(2 \times 10^{4}\right.$ cells/well for T3M4 migration, $4 \times 10^{4}$ cells/well for T3M4 invasion, $1.5 \times 10^{4}$ cells/well for SU86.86 and MiaPaCa-2 migration, $3 \times 10^{4}$ cells/well for SU86.86 and MiaPaCa- 2 invasion). DMEM or RPMI-1640 containing $10 \%$ fetal bovine 
serum was added to the lower chambers. After incubation at $37{ }^{\circ} \mathrm{C}$ and $5 \% \mathrm{CO}_{2}$ for $24 \mathrm{~h}$, cells that remained on the top of the filter were scrubbed off and cells that migrated or invaded to the lower surface were fixed in $90 \%$ ethyl alcohol and followed by hematoxylin-eosin staining for counting. Cell numbers were counted using light microscopy.

Western blotting. After transfection for 48 hours in 6-well plates $\left(5 \times 10^{5} \mathrm{cells} /\right.$ well $)$, total cellular proteins were extracted with RIPA buffer (Applygen, Beijing). Total protein $(100 \mu \mathrm{g})$ was separated on SDS-PAGE gel and then transferred to a polyvinylidene difluoride (PVDF) membrane (Millipore, Billerica, MA). After blocking with $5 \%$ non-fat milk at room temperature for 1 hour, the membrane was probed with primary antibodies $(1: 1000)$ overnight at $4{ }^{\circ} \mathrm{C}$. All primary antibodies (anti-YAP1:14074S; anti-EGFR:2322S; anti-b-catenin: 9562S; anti-b-actin:4970S) were bought from Cell Signaling Technology (Danvers, MA). The next day, incubate the membrane with a horseradish peroxidase-conjugated secondary antibody (C1308; 1:3000, Applygen, Beijing) at room temperature for 1 hour. Proteins were detected by ECL reagents (Millipore, Billerica, MA). Contents of RIPA lysis buffer: $50 \mathrm{mM}$ Tris- $\mathrm{HCl}$ (pH 7.4), $150 \mathrm{mM} \mathrm{NaCl}, 1 \% \mathrm{NP}-40$.

Statistical analysis. Continuous data were analyzed using the Student $t$ test or the Mann-Whitney U test. Categorical data were compared using a Pearson $\chi^{\bigotimes}$ test or Fisher exact test. The Kaplan-Meier method and Cox regression were used for survival analysis. All the statistical analyses were performed by SPSS v.13.0 (SPSS, Inc, Chicago, IL). A two- tailed $P<0.05$ was considered as statistical significance. All pictures were painted by Graphpad Prism 6.

\section{Results}

MiR-1285 is downregulated in gemcitabine-resistant AsPC-1 cells. AsPC-1-Gem and MiaPaCa-2-Gem cells are gemcitabine-resistance AsPC-1 and MiaPaCa- 2 cells, which could survive and proliferate in medium with high concentration of gemcitabine of $10 \mu \mathrm{M}$. We tested miR-1285 levels in AsPC-1-Gem and MiaPaCa-2-Gem cells compared with parent AsPC- 1 and MiaPaCa- 2 cells by Quantitative RT-PCR. Results showed that miR-1285 was significantly downregulated in AsPC-1-Gem and MiaPaCa-2 cells (Figure 1A). We also tested miR-1285 levels in 6 pancreatic cancer cell lines (Figure 1A). As miR-1285 expressed different in different cell lines, we chose T3M4 (lowest expression level), SU86.86 (highest expression level) and MiaPaCa-2 for followup experiments.

MiR-1285 impaired cell proliferation. As T3M4 cells had the lowest miR-1285 expression level compared to other cell lines, they were transfected with miR-1285 mimics or mimics control to verify the effect of miR-1285 up-regulation. SU86.86 cells were transfected with miR-1285 inhibitor or inhibitor control. MiaPaCa-2 cells were transfected both mimics and inhibitor, respectively. qRT-PCR demonstrated the transfect efficiency (Figure 1B). CCK8 assay results were showed in Figure 2. The proliferation rates of T3M4 were impaired after up-regulation of miR-1285 (Figure 2A). In contrast, down-regulation of miR-1285 increased the proliferation rate of SU86.86 (Figure 2A). In MiaPaCa-2 cells, the results were similar (Figure 2B). These data revealed that miR-1285 impaired cell proliferation in more than one cell lines.

MiR-1285 increased the sensitivity of PDAC cells to gemcitabine. We used growth inhibition assay to test the chemosensitivity of cells to gemcitabine. Data were collected with CCK8 assay. The number of survival cells after treated by gemcitabine for $48 \mathrm{~h}$ could reflect the chemoresistance of these cell. CCK8 assay was used to test the number of cells. The inhibition rate was calculated as $1-\frac{O D_{48 h}}{O D_{0 h}}$. The higher the inhibition rate,
A

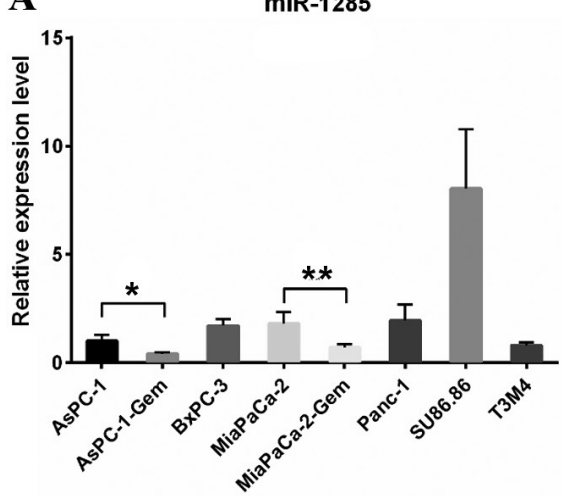

B

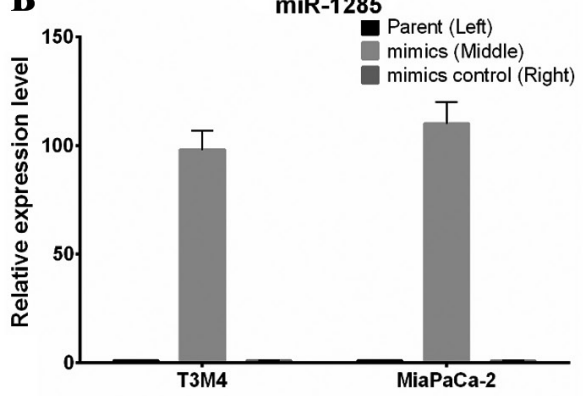

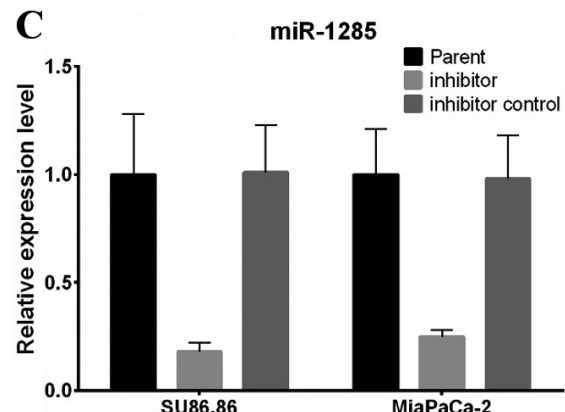

Figure 1. MiR-1285 levels in pancreatic cancer cell lines. (A) MiR-1285 was down-regulated in gemcitabine resistant AsPC-1 and MiaPaCa-2 cells compared to parent cells. MiR-1285 expression levels were relatively high in SU86.86 cells, while relatively low in T3M4 cells. We chose SU86.86, T3M4 and MiaPaCa-2 for further experiments. (B) qRT-PCR showed that after transfection with the miR-1285 mimics or the inhibitor, the expression of miR-1285 was significantly up-regulated or down-regulated, respectively. ${ }^{*} \mathrm{P}<0.05,{ }^{* *} \mathrm{P}<0.01,{ }^{* * *} \mathrm{P}<0.005$. 
A

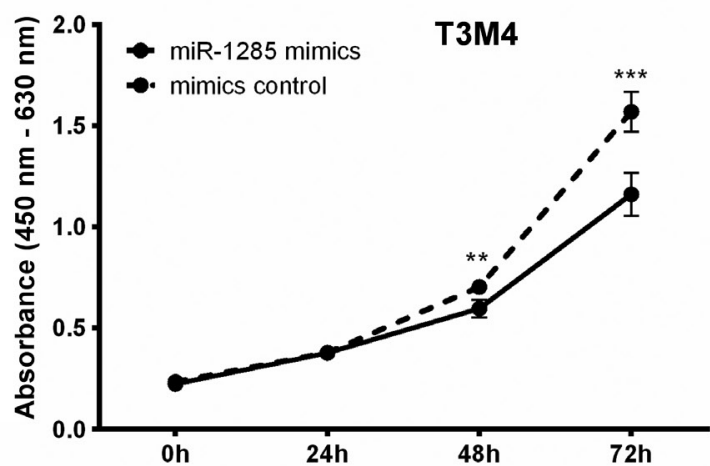

B

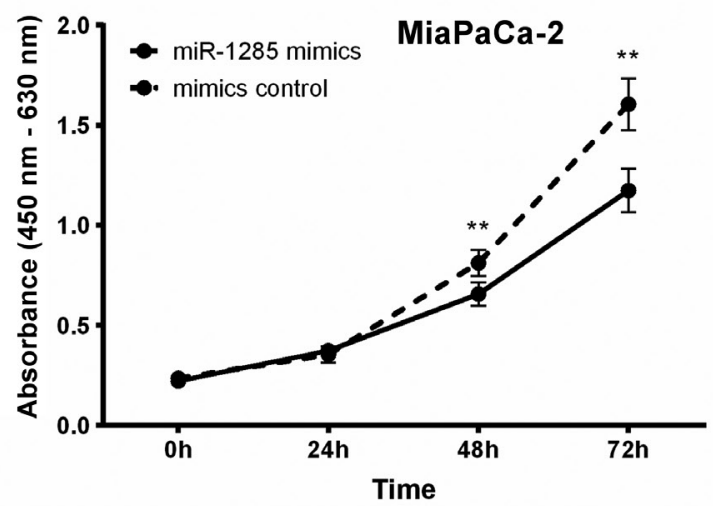

C

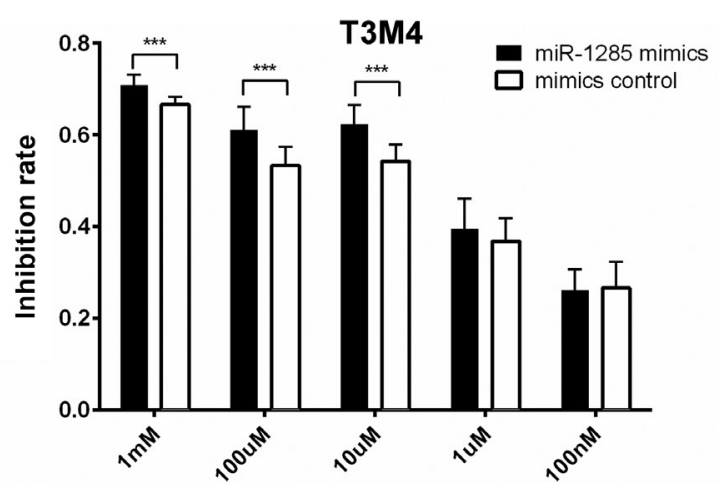

D

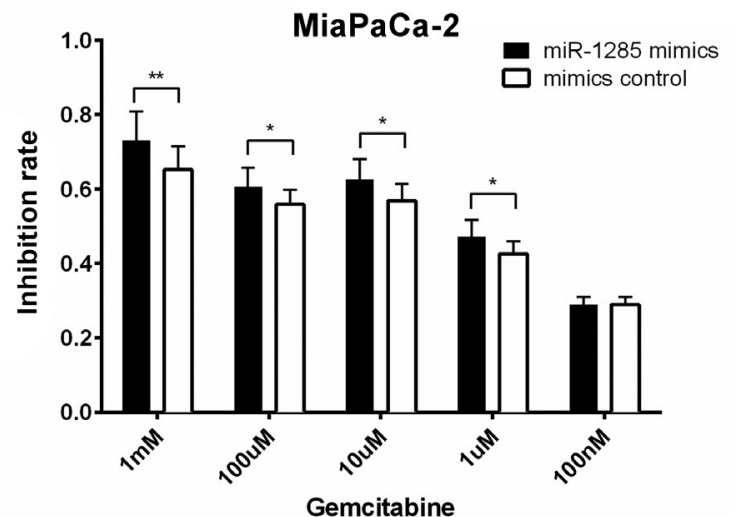

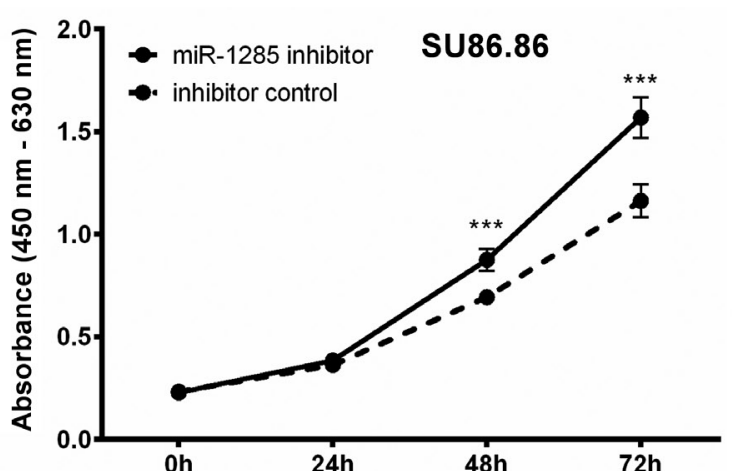
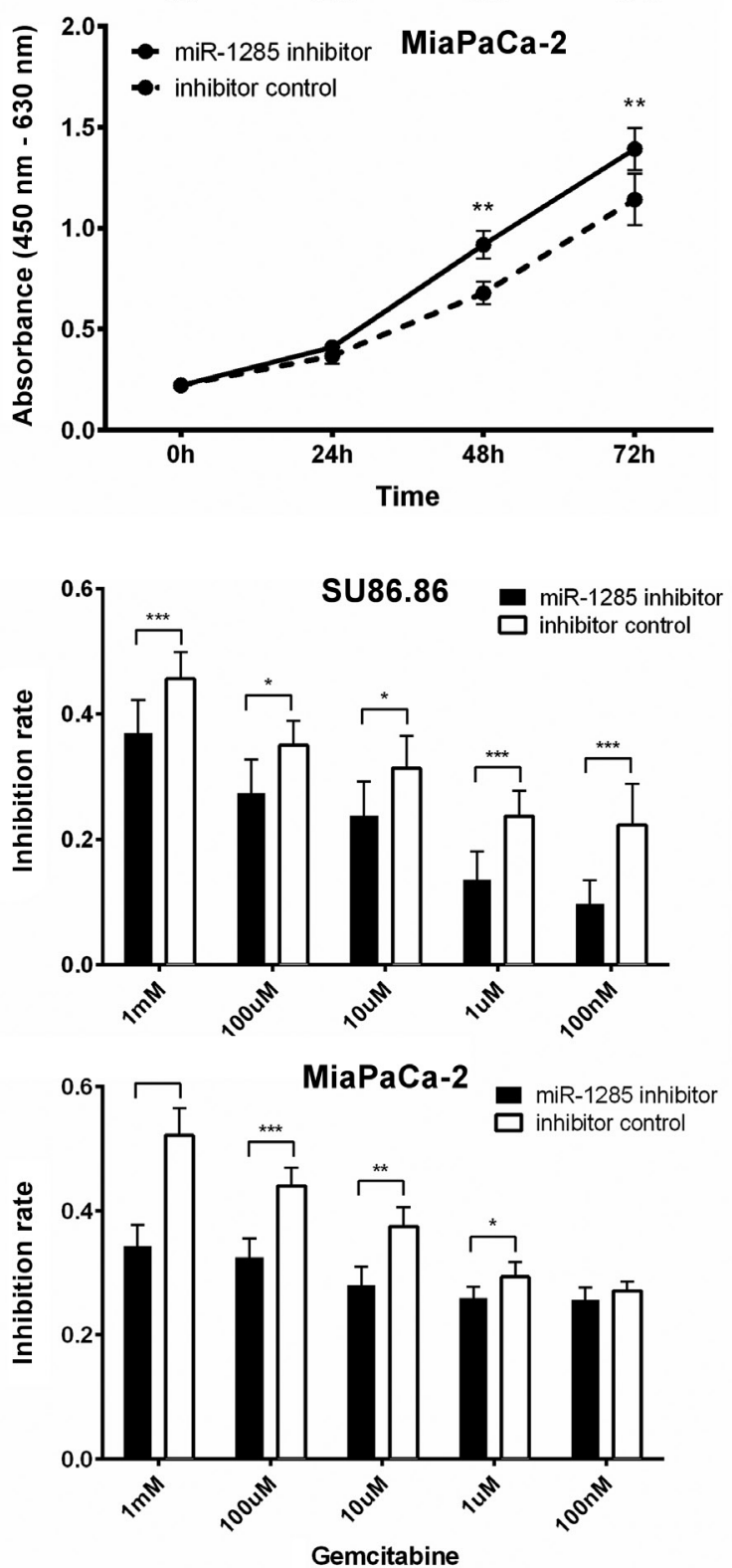

Figure 2. MiR-1285 impaired cell proliferation and increased sensitivity to gemcitabine. (A) A CCK-8 assay showed that miR-1285 over-expression inhibited the proliferation of T3M4 and MiaPaCa-2 cells.(B) The down-regulation of miR-1285 increased the proliferation of SU86.86 and MiaPaCa-2 cells. (C) A CCK8 assay showed that miR-1285 over-expression increased inhibition rate of gemcitabine, which meant increased sensitivity to gemcitabine treatment. (D) Downregulation of miR-1285 induced resistance to gemcitabine treatment. ${ }^{\star} \mathrm{P}<0.05,{ }^{* *} \mathrm{P}<0.01,{ }^{* * *} \mathrm{P}<0.005$. 
the more the chemosensitivity. Results showed that transfection of miR-1285 mimics in T3M4 cells decreased inhibition rate of gemcitabine, which meant miR-1285 significantly increased sensitivity to gemcitabine (Figure 2C). On the other site, downregulation of miR-1285 by transfection with inhibitor in SU86.86 cells increased resistance to gemcitabine treatment (Figure 2C). Similar results were seen in MiaPaCa-2 cells (Figure 2D).

MiR-1285 inhibited pancreatic cancer cell migration and invasion. Transwell assays were performed to explore whether miR-1285 could regulate cell migration and invasion of pancreatic cancer cell lines. The migrated or invaded cell numbers reflected cells' migratory or invasive ability, respectively. The over-expression of miR-1285 decreased the number of migrated T3M4 and MiaPaCa-2 cells (Figure 3A), while the knockdown of miR-1285 significantly increased the migrated SU86.86 and MiaPaCa-2 cell numbers (Figure 3B). The invasion assay acquired the similar results (Figure 3C, 3D). These data indicated that miR-1285 inhibited the migratory and invasive ability of different pancreatic cells.

MiR-1285 downregulated protein level of YAP1. Using TargetScan, Miranada and microRNA.org algorithms, YAP1 was predicted as a potential target of miR-1285 (Figure 4A). YAP1 mRNA had two binding sites to miR-1285 in its 3'UTR. To further confirm whether YAP1 was a regulated by miR-1285 or not, we used qRT-PCR and western blotting to verify the mRNA and protein level changes according to miR-1285 level changes, respectively. We found that miR-1285 didn't change YAP1 mRNA levels in T3M4 and SU86.86 (Figure 4B). However, up-regulation of miR-1285 decreased YAP1 protein level in T3M4 cell lines (Figure 4C). On the opposite site, the inhibition of miR-1285 showed the opposite effect (Figure 4C). We also detected the level of some key proteins in pancreatic cancer signaling pathway. When miR-1285 level was decreased, EGFR and $\beta$-catenin were also up-regulated together with YAP1 (Figure 4D). When miR-1285 was up-regulated, EGFR and $\beta$-catenin levels also decreased according to YAP1's changing trend (Figure 4D). As considerable researches and reviews reported the mutual promoted effects between YAP1 and EGFR or $\beta$-catenin for tumorigenesis [17-19], the protein level changing of EGFR and $\beta$-catenin further proved that miR-1285 may play a tumor suppressor role via downregulating YAP1 protein.

\section{Discussion}

Pancreatic ductal adenocarcinoma is a most deadly malignancy, whose main treatment strategy is chemotherapy. However, pancreatic cancer shows limited susceptibility to chemotherapy. The high rate of resistance to chemotherapeutic agent contributes to poor prognosis. Prior to this study, the biological functions of miR-1285 in pancreatic cancer have not been reported yet. Whether miR-1285 could regulate tumor cells' malignant behavior including chemosensitivity remains unclear. In this study, we demonstrated that miR-1285 was down-regulated in gemcitabine-resistant pancreatic cancer cells. Upregulation of miR-1285 suppressed cell proliferation, migration and invasion. Opposite effects were observed when miR-1285 was downregulated. MiR-1285 also regulated chemosensitivity of PDAC cells against gemcitabine. We also verified that miR-1285 negatively regulated YAP1 protein level, together with EGFR and $\beta$-catenin.

The regulatory roles of miR-1285 are still controversial. O.M. Niemoeller et al. [20] compared 1100 microRNA levels in six malignant cell lines before and after irradiation, finding that miR-1285 levels was upregulated to 2-3-fold after irradiation, indicating that miR-1285 may play a role in radiation induced cell death, proliferation, migration and metastasis. Liu J et al. [13] acquired a similar conclusion in a different way. They examined TACE therapeutic effectiveness-related microRNA levels systematically using miRNA Profiling Chips, finding that circulating miR-1285 levels are significantly higher in TACE-response group than TACE-nonresponse group. This result testifies our results that miR-1285 up-regulated in chemoresistant cells on the other side. What's more, in this article [13], quantitative analyses in additional HCC patients treated with TACE validated miR-1285 as candidate biomarkers for predicting prognosis after TACE. They also found that miR-1285 could directly repress the expression of oncogene JUN in HCC cells, indicating miR-1285 may act as a potential tumor suppressor. These two studies prove that miR-1285 may play important roles in regulation of cancer cells' response to chemotherapy and radiotherapy.

Hidaka. $\mathrm{H}$ et al. [14] discovered miR-1285 was significantly downregulated in renal cell carcinoma specimens by expression signatures. Further experiment suggested that miR-1285 significantly inhibited cancer cell proliferation, invasion, and migration by directly targeting transglutaminase 2 (TGM2). Up to now, studies of miR-1285 are only seen in these three kinds of solid tumors. Our results are supplement to the role of miR-1285 in solid cancer. However, our results seemed to conflict with one other research. Tian, S. et al [21] firstly identified miR-1285 as inhibitor of p53 in 2010, as there are two miR-1285 target sites in the 3' UTR of p53 mRNA. This seems to contradict with our results. However, this study only focuses on molecular biology level rather than cellular behavior level. Using seed rules, microRNAs can lead to a great diversity of phenotypic effects [22]. One microRNA may only lead to minor phenotype consequences. But when the system is sensitive enough, relatively weak repression by a miRNA could lead to substantial effects. There is a network between miRNAs and microRNAs. One single miRNA could repress hundreds of mRNAs while one mRNA may be repressed by multiple microRNAs [23]. Besides those, microRNA could lead to post-transcriptional gene silencing in three potential ways [24]. In addition to downregulating 
A

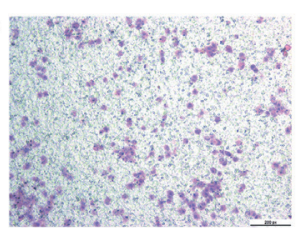

miR-1285 mimics

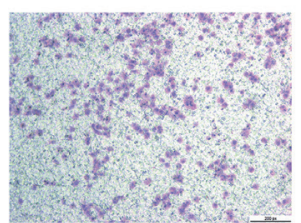

miR-1285 mimics

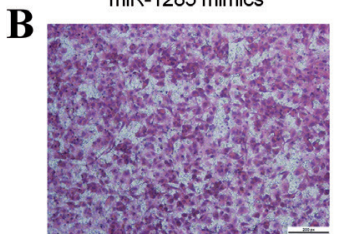

miR-1285 inhibitor

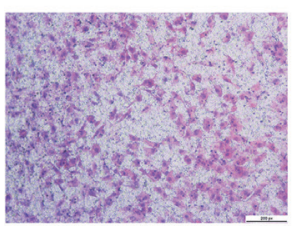

miR-1285 inhibitor

C

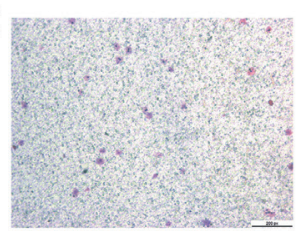

miR-1285 mimics

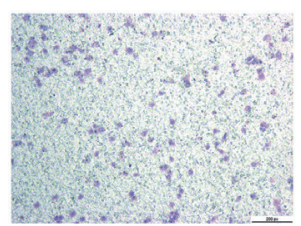

miR-1285 mimics

D

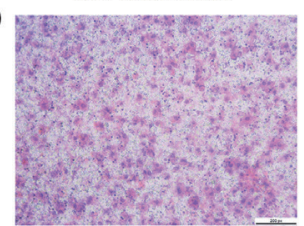

miR-1285 inhibitor

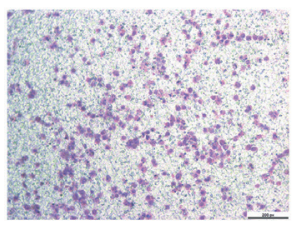

miR-1285 inhibitor

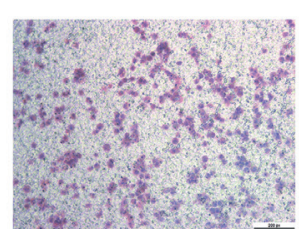

mimics control

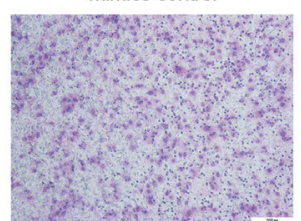

mimics control

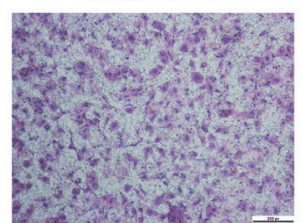

inhibitor control

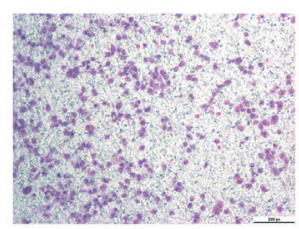

inhibitor contro

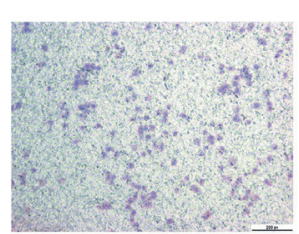

mimics control

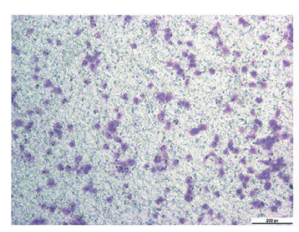

mimics control

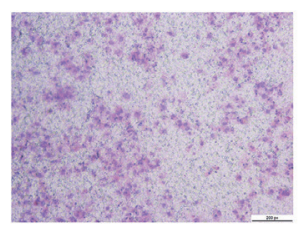

inhibitor control

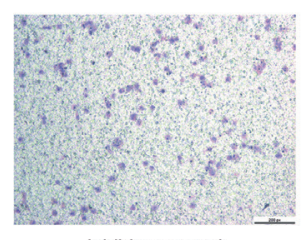

inhibitor contro

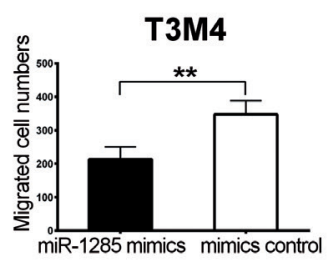

MiaPaCa-2

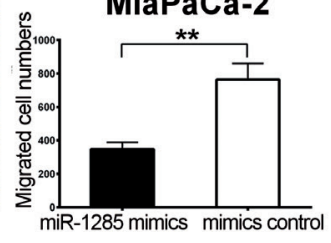

SU86.86

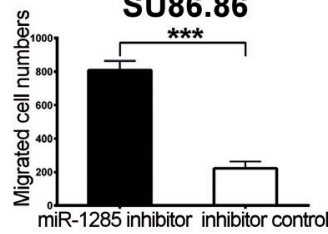

MiaPaCa-2
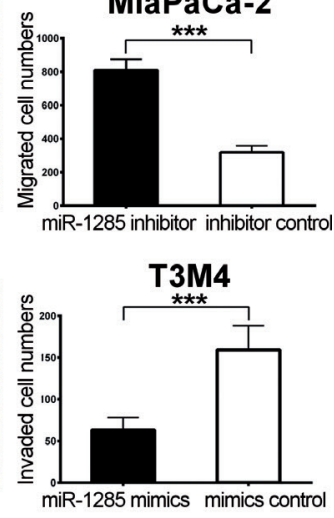

MiaPaCa-2

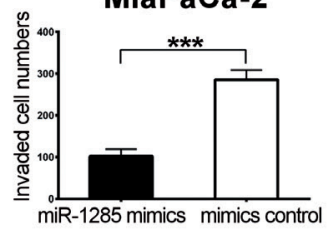

SU86.86

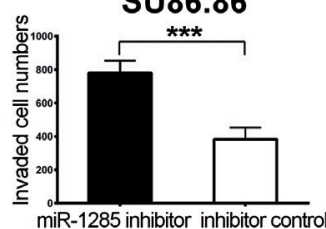

MiaPaCa-2

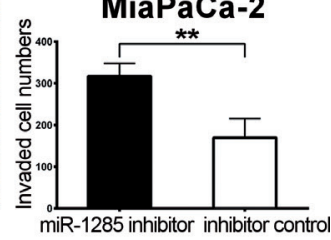

Figure 3. MiR-1285 inhibited pancreatic cancer cell migration and invasion. (A) A transwell assay showed that the over-expression of miR-1285 deterred cell migration. (B) MiR-1285 down-regulation enhanced cell migration. (C) Over-expression of miR-1285 inhibited cell invasion. (D) The down-regulation of miR-1285 promoted cell invasion. ${ }^{\star} \mathrm{P}<0.05,{ }^{* *} \mathrm{P}<0.01,{ }^{* * *} \mathrm{P}<0.005$. 
A

\begin{tabular}{|c|c|}
\hline $\begin{array}{l}\text { Position 312-318 of YAP1 3' UTR } \\
\text { hsa-miR-1285 }\end{array}$ & $\begin{array}{c}\text { 5, ...GGAUGCCAUUCCUUU--UGCCCAGU... } \\
\text { IIIII IIIIIIII } \\
\text { 3, } \\
\text { UCCAGAGUGAAACAACGGGUCU }\end{array}$ \\
\hline $\begin{array}{l}\text { Position 2525-2531 of YAP1 3' UTR } \\
\text { hsa-miR-1285 }\end{array}$ & $\begin{array}{c}\text {...CCCUCAACUUUAAUUUGCCCAGU... } \\
\text { III }|| \text { I I I I I I } \\
\text { UCCAGAGUGAAACA--ACGGGUCU }\end{array}$ \\
\hline
\end{tabular}

B

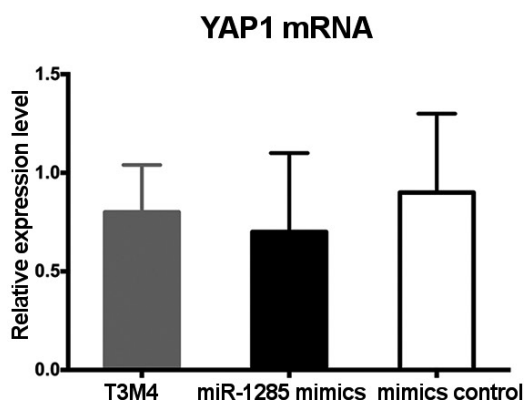

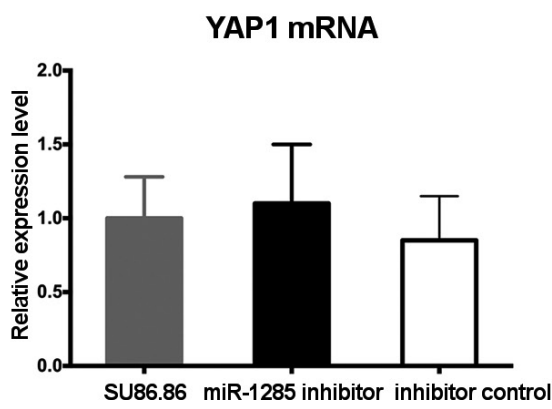

C

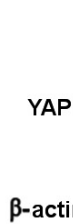

D

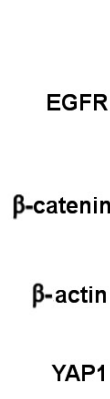

T3M4

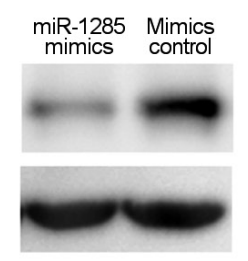

T3M4

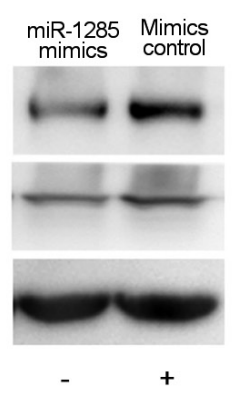

SU86.86

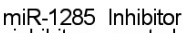
miR-1285 Inhibitor
inhibitor control
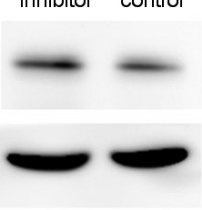

SU86.86

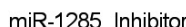
inhibitor control

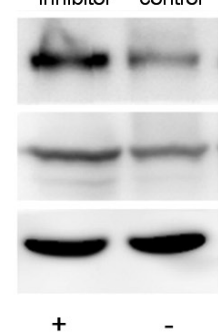

Figure 4. YAP1 is a target of miR-1285. (A) Two miR-1285 binding sites within the 3' -UTR of YAP1was predicted by TargetScan. (B) Transfection with the miR-1285 mimics or the inhibitor did not change the mRNA expression of YAP1. (C) The protein levels of YAP1 were detected by western blot after T3M4 and SU86.86 cells were transfected with the miR-1178 mimics or the inhibitor, respectively. $\beta$-actin was used as an internal control. (D) The protein levels of EGFR and beta-catenin showed the similar variation tendency to YAP1.

mRNA levels, a few microRNAs could regulate human gene expression in a transcriptional manner [25] or by enhancing translation [26]. All these mechanisms could overlap in one cell or one protein. We believe that the regulating mechanism of miR-1285 needs further research. Contrary to our results, recent literature shows that miR-1285 serum level seems to be upregulated in pancreatic cancer patients [27]. There are two possible explanations of the discrepancies. Firstly, the role of miR-1285 in diagnosis hasn't been validated as a diagnostic marker in the article. Secondly, tumorigenesis is the consequence of sequential expression and interaction between oncogenes and tumor suppressors. Tumor suppressive microRNAs could be upregulated in serum. For example, miR-200 family is known as tumor suppressors in many cancers $[28,29]$. However, other researchers found that serum expression of $\mathrm{miR}-200 \mathrm{a}$ much greater in cancer cells in comparison with matched normal controls. MiR-200 family seems to be associated with the more aggressive tumor progression and be recognized as reliable prognostic markers in cancer patients [30].

Our findings also give clues with respect to miRNAstargeted pancreatic cancer treatment. YAP1 is a well-known oncoprotein [16. 31]. In pancreatic cancer, YAP1 activation enables bypass of oncogenic K-Ras addiction in pancreatic cancer [32]. When oncogenic K-Ras slumbers, YAP1 is activated and leads to a more aggressive phenotype in mouse model [33]. These studies suggest that silencing of YAP1 activity might be beneficial in cancer prevention and treatment. Further research elucidating the exact mechanisms of miRNA-1285 function is needed.

In general, in vitro data is not sufficient for drug developing for pancreatic cancer. In our future study, we will elucidate the exact regulatory mechanisms of miRNA-1285 in vivo. In addition, we will evaluate the correlation between miR-1285 tissue or serum levels and clinicopathological characteristics and the drug efficacy of pancreatic cancer.

In conclusion, this study demonstrated that miR-1285 suppressed cell proliferation, migration and invasion, as well as increased the sensitivity of PDAC cells to gemcitabine in vitro. Our data suggests that miR-1285 may act as a tumor suppressor of pancreatic cancer. To our knowledge, this study is the first to elucidate the function of miR-1285 in pancreatic cancer. We also verified that miR-1285 negatively regulated YAP1 protein level, together with EGFR and $\beta$-catenin. Our results also suggest that miR-1285 might act as a novel therapeutic target for miRNA-based therapy in pancreatic cancer in the future.

Acknowledgements: This study was supported by grants from the National Natural Science Foundation of China (No.81272484 \& No. 81472327), Beijing Natural Science Foundation (No. 7132179), Major State Basic Research Development Program of China (973 Program, No. 2014CB542300). No potential conflicts of interest were disclosed. 


\section{References}

[1] SIEGEL RL, MILLER KD, JEMAL A. Cancer statistics, 2015. CA Cancer J Clin 2015; 65: 5-29. https://doi.org/10.3322/ caac. 21254

[2] RYAN DP, HONG TS, BARDEESY N. Pancreatic adenocarcinoma. N Engl J Med 2014; 371: 1039-1049. https://doi. org/10.1056/NEJMra1404198

[3] BURRIS HA, 3RD, MOORE MJ, ANDERSEN J, GREEN MR, ROTHENBERG ML ET AL. Improvements in survival and clinical benefit with gemcitabine as first-line therapy for patients with advanced pancreas cancer: a randomized trial. J Clin Oncol 1997; 15: 2403-2413.

[4] OETTLE H, NEUHAUS P, HOCHHAUS A, HARTMANN JT, GELLERT K et al. Adjuvant chemotherapy with gemcitabine and long-term outcomes among patients with resected pancreatic cancer: the CONKO-001 randomized trial. JAMA 2013; 310: 1473-1481. https://doi.org/10.1001/ jama.2013.279201

[5] MOORE MJ, GOLDSTEIN D, HAMM J, FIGER A, HECHT $\mathrm{JR}$ et al. Erlotinib plus gemcitabine compared with gemcitabine alone in patients with advanced pancreatic cancer: a phase III trial of the National Cancer Institute of Canada Clinical Trials Group. J Clin Oncol 2007; 25: 1960-1966. https://doi.org/10.1200/JCO.2006.07.9525

[6] VON HOFF DD, ERVIN T, ARENA FP, CHIOREAN EG, INFANTE $J$ et al. Increased survival in pancreatic cancer with nab-paclitaxel plus gemcitabine. N Engl J Med 2013; 369: 1691-1703. https://doi.org/10.1056/NEJMoa1304369

[7] STAN SD, SINGH SV, BRAND RE. Chemoprevention strategies for pancreatic cancer. Nat Rev Gastroenterol Hepatol 2010; 7: 347-356. https://doi.org/10.1038/nrgastro. 2010.61

[8] NCCN guidelines of PDAC. Version 2. 2015. https://www. nccn.org/professionals/physician_gls/pdf/pancreatic.pdf, accessed on Dec 02, 2016.

[9] CONROY T, DESSEIGNE F, YCHOU M, BOUCHE O, GUIMBAUD R et al. FOLFIRINOX versus gemcitabine for metastatic pancreatic cancer. N Engl J Med 2011; 364: 1817-1825. https:// doi.org/10.1056/NEJMoa1011923

[10] CALIN GA, CROCE CM. MicroRNA signatures in human cancers. Nat Rev Cancer 2006; 6: 857-866. https://doi. org/10.1038/nrc1997

[11] DONG W, LI B, WANG Z, ZHANG Z, WANG J. Clinical significance of microRNA-24 expression in esophageal squamous cell carcinoma. Neoplasma 2015; 62: 250-258. https:// doi.org/10.4149/neo 2015030

[12] LIU N, ZHONG L, ZENG J, ZHANG X, YANG Q et al. Upregulation of microRNA-200a associates with tumor proliferation, CSCs phenotype and chemosensitivity in ovarian cancer. Neoplasma 2015; 62: 550-559. https://doi.org/10.4149/ neo $2015 \quad 066$

[13] LIU J, YAN J, ZHOU C, MA Q, JIN Q et al. miR-1285-3p acts as a potential tumor suppressor miRNA via downregulating JUN expression in hepatocellular carcinoma. Tumour Biol 2015; 36: 219-225. https://doi.org/10.1007/s13277-014$\underline{2622-5}$
[14] HIDAKA H, SEKI N, YOSHINO H, YAMASAKI T, YAMADA $Y$ et al. Tumor suppressive microRNA-1285 regulates novel molecular targets: aberrant expression and functional significance in renal cell carcinoma. Oncotarget 2012; 3: 44-57.

[15] PAN D. The hippo signaling pathway in development and cancer. Dev Cell 2010; 19: 491-505. https://doi.org/10.1016/j. devcel.2010.09.011

[16] HARVEY KF, ZHANG X, THOMAS DM. The Hippo pathway and human cancer. Nat Rev Cancer 2013; 13: 246-257. https:// doi.org/10.1038/nrc3458

[17] ROSENBLUH J, NIJHAWAN D, COX AG, LI X, NEAL JT et al. beta-Catenin-driven cancers require a YAP1 transcriptional complex for survival and tumorigenesis. Cell 2012; 151: 1457-1473. https://doi.org/10.1016/j.cell.2012.11.026

[18] AVRUCH J, ZHOU D, BARDEESY N. YAP oncogene overexpression supercharges colon cancer proliferation. Cell Cycle 2012; 11: 1090-1096. https://doi.org/10.4161/ cc.11.6.19453

[19] SONG S, HONJO S, JIN J, CHANG SS, SCOTT AW et al. The Hippo Coactivator YAP1 Mediates EGFR Overexpression and Confers Chemoresistance in Esophageal Cancer. Clin Cancer Res 2015; 21: 2580-2590. https://doi.org/10.1158/1078-0432. CCR-14-2191

[20] NIEMOELLER OM, NIYAZI M, CORRADINI S, ZEHENTMAYR F, LI M et al. MicroRNA expression profiles in human cancer cells after ionizing radiation. Radiat Oncol 2011; 6: 29. https://doi.org/10.1186/1748-717X-6-29

[21] TIAN S, HUANG S, WU S, GUO W, LI J et al. MicroRNA-1285 inhibits the expression of p53 by directly targeting its $3^{\text {c }}$ untranslated region. Biochem Biophys Res Commun 2010; 396 : 435-439. https://doi.org/10.1016/j.bbrc.2010.04.112

[22] FLYNT AS, LAI EC. Biological principles of microRNAmediated regulation: shared themes amid diversity. Nat Rev Genetics 2008; 9: 831-842. https://doi.org/10.1038/ nrg2455

[23] SELBACH M, SCHWANHAUSSER B, THIERFELDER N, FANG Z, KHANIN R et al. Widespread changes in protein synthesis induced by microRNAs. Nature 2008; 455: 58-63. https://doi.org/10.1038/nature07228

[24] LIZ, RANA TM. Therapeutic targeting of microRNAs: current status and future challenges. Nat Rev Drug Discov 2014; 13: 622-638. https://doi.org/10.1038/nrd4359

[25] TAN Y, ZHANG B, WU T, SKOGERBO G, ZHU X et al. Transcriptional inhibiton of Hoxd4 expression by miRNA10a in human breast cancer cells. BMC Mol Biol 2009; 10: 12. https://doi.org/10.1186/1471-2199-10-12

[26] OROM UA, NIELSEN FC, LUND AH. MicroRNA-10a binds the 5'UTR of ribosomal protein mRNAs and enhances their translation. Mol Cell 2008; 30: 460-471. https://doi. org/10.1016/j.molcel.2008.05.001

[27] XU J, CAO Z, LIU W, YOU L, ZHOU L et al. Plasma miRNAs Effectively Distinguish Patients With Pancreatic Cancer From Controls: A Multicenter Study. Ann Surg 2016; 263: 1173-1179. https://doi.org/10.1097/SLA.0000000000001345

[28] YE F, TANG H, LIU Q, XIE X, WU M et al. miR-200b as a prognostic factor in breast cancer targets multiple mem- 
bers of RAB family. J Transl Med 2014; 12: 17. https://doi. org/10.1186/1479-5876-12-17

[29] WILLIAMS LV, VELICEASA D, VINOKOUR E, VOLPERT OV. miR-200b inhibits prostate cancer EMT, growth and metastasis. PloS One 2013; 8: e83991. https://doi.org/10.1371/ journal.pone.0083991

[30] ZUBERI M, MIR R, DAS J, AHMAD I, JAVID J et al. Expression of serum miR-200a, miR-200b, and miR-200c as candidate biomarkers in epithelial ovarian cancer and their association with clinicopathological features. Clin Transl Oncol 2015; 17: 779-787. https://doi.org/10.1007/s12094-015-1303-1
[31] JOHNSON R, HALDER G. The two faces of Hippo: targeting the Hippo pathway for regenerative medicine and cancer treatment. Nat Rev Drug Discov 2014; 13: 63-79. https://doi. org/10.1038/nrd4161

[32] GRETEN FR. YAP1 takes over when oncogenic K-Ras slumbers. Cell 2014; 158: 11-12. https://doi.org/10.1016/j. cell.2014.06.021

[33] KAPOOR A, YAO W, YING H, HUA S, LIEWEN A et al. Yap1 activation enables bypass of oncogenic Kras addiction in pancreatic cancer. Cell 2014; 158: 185-197. https://doi. org/10.1016/j.cell.2014.06.003 